\title{
A Cost of Illness Analysis of Hepatitis B Virus Infection in Greece
}

\author{
Athanasakis $\mathrm{K}^{1^{*}}$, Pliarchopoulou $\mathrm{F}^{2}$, Reppas $\mathrm{L}^{3}$, Brountzos $\mathrm{E}^{3}$, Filippiadis $\mathrm{D}^{3}$, Foukas $\mathrm{P}^{4}$, \\ Sidiropoulos $\mathrm{O}^{2}$, Kyriopoulos $\mathrm{J}^{1}$, Boumpas $\mathrm{D}^{2}$ and Chounta $\mathrm{A}^{2}$ \\ ${ }^{1}$ Department of Health Economics, National School of Public Health, Athens \\ 2 4th Department of Internal Medicine, "Attikon" Hospital, National and Kapodistrian University of Athens \\ ${ }^{3}$ 2nd Department of Radiology, "Attikon" Hospital, National and Kapodistrian University of Athens \\ ${ }^{4}$ 2nd Department of Pathology, "Attikon" Hospital, National and Kapodistrian University of Athens
}

\begin{abstract}
Received: March 17, 2017; Accepted: May 24, 2017; Published: July 10, 2017
*Corresponding author:Kostas Athanasakis, Research Fellow, Department of Health Economics, National School of Public Health, Athens, 196 Alexandras Avenue, 11521, Athens, Greece, Tel no: +302132010242; Fax: +302106449571; E-mail: kathanasakis@esdy.edu.gr
\end{abstract}

\begin{abstract}
Background/Objective: Although often called a "silent disease", HBV entails a significant disease and financial burden for the health system. An attempt to provide detailed estimates of the direct cost of this - rather neglected - disease in Greece comprised the objective of this study.
\end{abstract}

Methods: The medical records of 254 patients diagnosed with HBV and monitored in the Hepatology Unit of the 'Attikon' university hospital provided the basis for the analysis. Detailed resource use data (physician visits, medications, labwork, and hospitalizations) were derived from the patients' records for a retrospective 12 month period, before their most recent visit to the clinic. Calculations followed a third-party payer perspective, according to official prices and tariffs, and are expressed in year 2015 Euros.

Results: $59 \%$ of patients were male with an average age of 50.7 years and an average duration since diagnosis of 13.2 years. Overall, the average annual cost per patient was estimated at 1,822.5 (s.d.: 2,236.5) and 886.7 (s.d.: 703.1.5) Euros including and excluding HBV medications, respectively. Average annual cost per patient according to stage of fibrosis was 1,628.56 (840.49), 2,546.1 (1,068.2) and $4.764,8(1,590.2)$ Euros for patients with no fibrosis/no cirrhosis, fibrosis present and cirrhosis present, respectively (numbers in brackets represent the cost without HBV medications). Medications accounted for $51 \%$ of total cost, followed by laboratory work(38\%) and hospitalizations (9\%).

Conclusions: The cost of HBV is substantial and increases up to $300 \%$, as the disease progresses. The findings of this study suggest that HBV is a costly disease, especially when taking into account its prevalence.

Keywords: Cost-of-illness; Hepatitis B virus; Economic evaluation; Direct cost

\section{Introduction}

Despite the widely acknowledged progress of the last decades in the understanding and management of liver disease and the availability of both treatment as well as preventive alternatives, viral hepatitis remains a key public health (and health policy) issue [1]. It is estimated that $1.8 \%$ of the adult population - or 13.3 million persons - in the WHO-EURO Region suffer from chronic HBV [2]; approximately 36,000 persons die each year as a consequence of the disease, whereas a significant proportion of patients experience severe complications, such as cirrhosis and hepatocellular carcinoma. Inevitably, the latter result to increases in the total burden of disease and, consequently, to a significant pressure on healthcare budgets.

The recent developments in the therapeutic armamentarium of HCV and the curative potential of the newgeneration medications have attracted almost the entirety of research efforts in the clinical, epidemiological and health policy domain of hepatitis, leaving HBV "in the shadows". As a result, although the two diseases appear comparable in terms of disease burden and mortality [3], HBV remains under-investigated, especially regarding its health-policy and -economics implications. Indicatively, the last "series" of cost-of-illness papers in HBV, i.e. the type of analyses that are published today more frequently than ever in HCV, can be traced as back as 2004, whereas a paucity of data more or less remains ever since.

In light of the above, and bearing in mind the significant prevalence of $\mathrm{HBV}$ in Greece, the severe constraints in the availability of health resources in the country and the absence of an up-to-date previous estimation, the objective of this study was to conduct a cost-of-illness analysis for HBV in Greece, under the third party payer perspective.

\section{Methods}

The present analysis is a microcosting study based on a retrospective review of the medical records of a sample of patients diagnosed with HBV in Greece. The primary data for the analyses are sourced from the medical records of 254 patients diagnosed with HBV and monitored in the Hepatology Unit of 4th Department of Internal Medicine in "Attikon" University Hospital. To avoid selection bias, investigators identified potentially 
eligible patients from consecutive visits in the clinic visit register Starting with the patient with the most recent visit, patients with consecutive visits (backwards in time) were screened. Inclusion criteria were a) age $>18$ yrs b) diagnosis of $\mathrm{HBV}$ c) being followedup by the center for at least 1 year prior to the most recent visit. For the purposes of the study, an approval from the hospital's Bioethics committee was obtained (Nr6/07.04.15).

The data were extracted from the patient's medical file starting from the inclusion date (visit) and moving backwards for a period of 365 days. The collected data comprised patient demographic and clinical characteristics, severity of disease (stage of fibrosis), comorbidities, and health-resource utilization (medications, laboratory tests, biopsies, imaging tests, physician visits, and hospitalizations).

The annual direct medical cost was calculated taking into account the resources used and the unitary costs at the local level from the third party payer's perspective (social insurance). Cost of medications was taken from the official drug Price Bulletin. The costs of inpatient stays were calculated according to the Greek DRG system. The cost of biopsies, laboratory tests as well as cost of visits to specialists are based on official tariffs. All unit costs and cost calculations correspond to year 2015 values

\section{Results}

The study sample was predominantly male (59\%) with an average age of 50.7 (s.d. 16.2) years and an average duration since initial diagnosis of 13.2 years (s.d. 9.45). The majority of patients $(89.7 \%)$ had no fibrosis or cirrhosis present (F0-F2), $5.5 \%$ had evidence of fibrosis (F3) and the remaining $4.7 \%$ had laboratory-confirmed cirrhosis (F4).

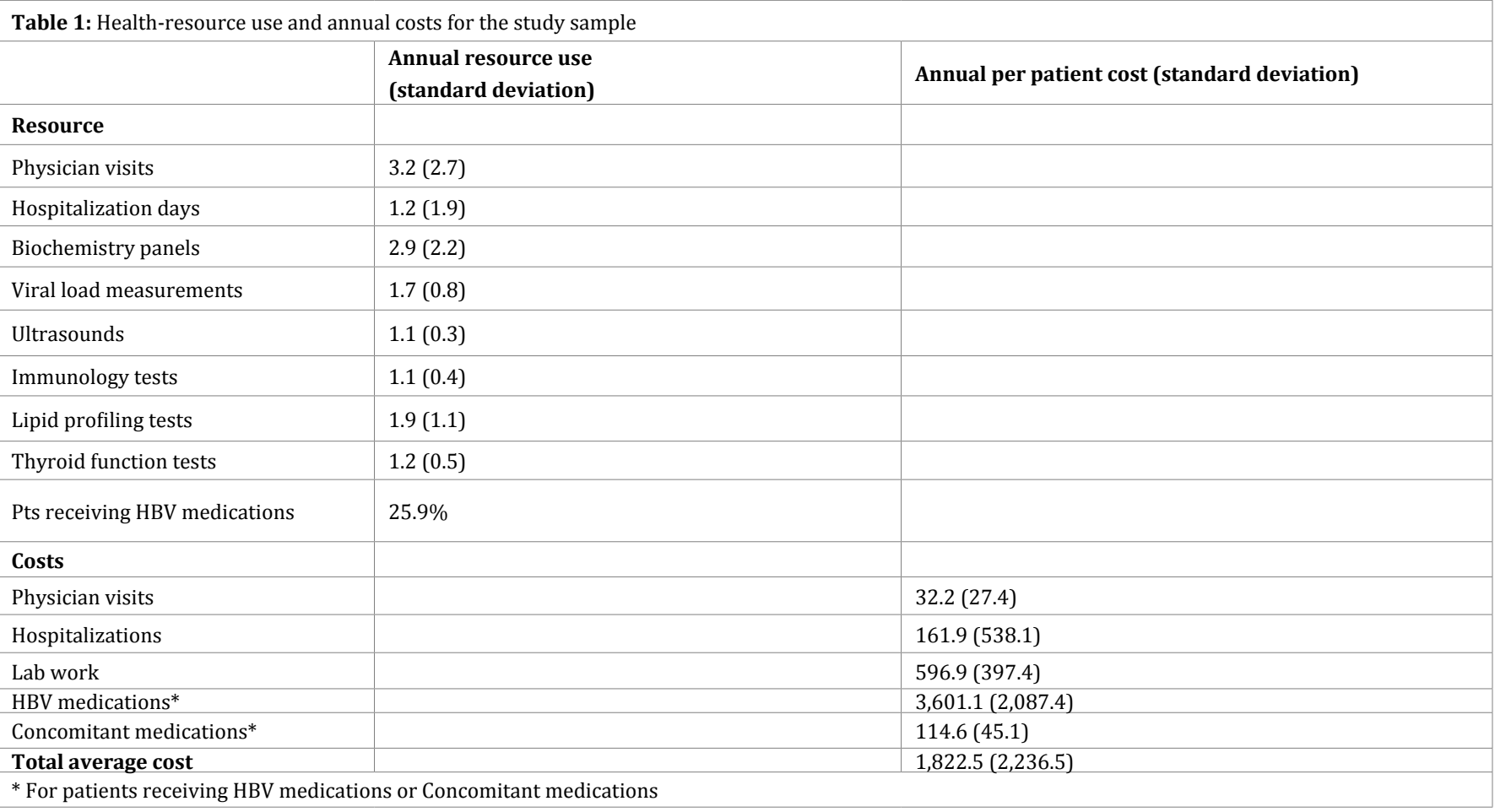

Table 1 provides the average annual resource use (for the most commonly used healthcare resources) and the average annual cost per patient in the study sample. Overall and for all patients included in the analysis, regardless of disease severity, the average annual cost per patient was estimated at 1,822.5 (s.d.: 2,236.5) and 886.7 (s.d.: 703.1.5) Euros including and excluding HBV medications, respectively. Medications accounted for $51 \%$ of total cost, followed by labwork (38\%) and hospitalizations (9\%). Average annual cost per patient according to stage of fibrosis was 1,628.56 (840.49), 2,546.1 (1,068.2) and 4.764,8 (1,590.2) Euros for patients with no fibrosis/no cirrhosis (F0-F2), fibrosis present (F3) and cirrhosis present (F4), respectively (numbers in brackets represent the cost without HBV medications). Differences were statistically significant (at the 0.05 level) among the groups of patients when the presence of cirrhosis was taken into account.

\section{Discussion}

In light of the scarcity of data on the financial burden of HBV for healthcare systems internationally, the present study attempted to provide insights based on local data for Greece. The results are in accordance with the existing international literature in terms of the increasing trend in the annual costs per patient as the disease progresses - similar findings have been reported for Germany, the US and Australia [4,5,6]. However, the absolute (nominal) estimates between Greece and other countries do differ and are, in fact, lower, probably as a result of lower prices for pharmaceuticals in Greece, compared to other 
EU countries, lower tariffs for inpatient care, laboratory work and physician consultations and lower wage levels, especially in the present times of austerity, compared to countries like Australia or Germany [7].

As with any study of this kind, the present one has a number of limitations that should be taken into account during the interpretation of the results. In specific, the fact that this is a single-center study that is undertaken in the premises of a specialized clinic could affect the generalizability of the results, - although according to the current status quo of HBV in Greece, patient monitoring is usually performed in specialized units of the system. Another limitation of the study is the fact that it only records and calculates direct costs (due to the third party payer perspective that is adopted in the analysis). This limits the results only on costs that are payed by the system and not by the patients themselves or the society - the so called "indirect costs". In this sense, the calculations are most probably conservative.

\section{Conclusions}

The present analysis suggests that HBV is a costly disease for Greece; at a per patient average annual direct cost of 1,822.5 Euros - a cost that increases almost three-fold in the presence of cirrhosis - the total expenditure attibutable to the disease for an estimated prevalence of $1.05 \%$ for known cases would be 211 million Euros or $2.4 \%$ of the country's total public expenditure on health [8].

\section{Conflict of Interest}

The authors declare no potential conflicts of interest with relation to this work

\section{References}

1. Papatheodoridis G, Thomas HC, Golna C, Bernardi M, Carballo M, Cornberg $\mathrm{M}$, et al. Addressing barriers to the prevention, diagnosis and treatment of hepatitis $\mathrm{B}$ and $\mathrm{C}$ in the face of persisting fiscal constraints in Europe: report from a high level conference. J Viral Hepat. 2016;Suppl 1:1-12. doi: 10.1111/jvh.12493

2. World Health Organization (WHO). Hepatitis B. Fact Sheet No 204. 2014.

3. Stanaway JD, Flaxman AD, Naghavi M, Christina Fitzmaurice, Theo Vos, Ibrahim Abubakar, et al The global burden of viral hepatitis from 1990 to 2013: findings from the Global Burden of Disease Study 2013. Lancet. 2016;388(10049):1081-1088.

4. Harbarth S, Szucs T, Berger K, Jilg W. The economic burden of hepatitis B in Germany. Eur J Epidemiol. 2000;16(2):173-177.

5. Lee TA, Veenstra DL, Iloeje UH, Sullivan SD. Cost of chronic hepatitis B infection in the United States. J Clin Gastroenterol. 2004;38(10 Suppl 3):S144-147.

6. Butler JR, Pianko S, Korda RJ, Nguyen S, Gow PJ, Roberts SK, Strasser SI, Sievert $\mathrm{W}$. The direct cost of managing patients with chronic hepatitis B infection in Australia. J Clin Gastroenterol. 2004;38(10 Suppl 3):S187-192.

7. Athanasakis K, Kyriopoulos II, Sideris M, Rentzos M, Evdokimidis J, Kyriopoulos J. Investigating the economic burden of ALS in Greece: a cost-of-illness approach. Amyotroph Lateral Scler Frontotemporal Degener. 2015;16(1-2):63-64. doi: 10.3109/21678421.2014.932384

8. Papatheodoridis G, Sypsa V, Kantzanou M, Nikolakopoulos I, Hatzakis A. Estimating the treatment cascade of chronic hepatitis B and C in Greece using a telephone survey. J Viral Hepat. 2015;22(4):409-415. doi: $10.1111 /$ jvh.12314 